\title{
NEOPLASIA PAPILAR CÍSTICA DO PÂNCREAS
}

\section{PAPYLLARY CYSTIC TUMOR OF THE PANCREAS}

\author{
Francisco Edilson Leite Pinto Junior, TCBC-RN ${ }^{1}$ \\ Marcus Vinícius de Moraes $^{2}$ \\ Álisson Giovani Freitas de Oliveira ${ }^{3}$
}

\section{INTRODUÇÃO}

O tumor sólido-cístico do pâncreas, descrito inicialmente por Frantz, em 1954 ${ }^{1}$ é uma neoplasia rara que acomete geralmente pacientes jovens do sexo feminino, cujo tamanho varia entre sete a $20 \mathrm{~cm}$ e se estende ao longo da cabeça, corpo e cauda do pâncreas ${ }^{2,3}$. Tem sido conhecido por diversas denominações: neoplasia epitelial sólida papilar, neoplasia papilar cística, carcinoma papilar-cístico, neoplasia papilífera de baixo grau e tumor de Frantz ${ }^{2,3}$.

Manifesta-se clinicamente através de massa abdominal assintomática e se comporta como tumor benigno ou de baixa malignidade, tendo excelente prognóstico com a ressecção cirúrgica completa . Este comportamento evolutivo contrasta com a apresentação do adenocarcinoma de pâncreas que, no momento do diagnóstico, quase sempre significa câncer localmente avançado e com metástase a distância ${ }^{2}$.

O objetivo deste trabalho é relatar dois casos de tumor de Frantz, discutindo os aspectos referentes ao diagnóstico, patologia, prognóstico e tratamento desta neoplasia.

\section{RELATO DOS CASOS}

\section{Caso 1}

Paciente do sexo feminino, 26 anos, apresentando quadro clínico compatível com colecistite aguda. Durante investigação ultra-sonográfica foi evidenciada, além da colelitíase e inflamação da vesícula biliar, um tumor sólido-cístico em corpo e cauda do pâncreas de aproximadamente $10 \times 8 \mathrm{~cm}$ de tamanho. Foi orientada em outro serviço a submeter-se à colecistectomia por via laparoscópica, observando-se clinicamente a lesão pancreática. Dez meses após esta conduta, passou a apresentar dor em hipo- côndrio esquerdo de moderada intensidade. Procurou nosso serviço sendo submetida à tomografia computadorizada (TC) e indicado tratamento cirúrgico (Figuras 1A e 1B). Realizada pancreatectomia corpo-caudal associada à esplenectomia, através de incisão subcostal bilateral. O estudo anatomopatológico revelou tumor papilífero sólido-cístico do pâncreas. A paciente recebeu alta no sexto dia de pós-operatório (DPO), encontrando-se assintomática e sem evidência da doença após 42 meses.

\section{Caso 2}

Paciente do sexo feminino, 31 anos, apresentando dor em hipocôndrio esquerdo há 10 anos, de moderada intensidade, sem irradiação e/ou relação com a alimentação. Negava icterícia, anorexia e perda de peso. Ao exame físico, palpava-se massa em hipocôndrio e flanco esquerdos, com cerca de $8 \mathrm{~cm}$, móvel e de consistência elástica-firme. A TC evidenciou lesão expansiva sólida-cística em topografia do corpo e cauda do pâncreas, de aproximadamente $18 \times 12 \mathrm{~cm}$ (Figura 2-A). Foi submetida à laparotomia exploradora, através de incisão subcostal bilateral, sendo realizadas pancreatectomia corpo-caudal e esplenectomia (Figura 2-B). O estudo anatomopatológico revelou tumor papilífero sólido-cístico do pâncreas. Recebeu alta no 7o DPO, encontrando-se assintomática e sem evidência de doença após 13 meses.

\section{DISCUSSÃO}

Desde seu primeiro relato por Frantz, em 1954, ainda pairam dúvidas sobre a origem histológica epitelial (se ductal ou acinar) da neoplasia sólido-cística do pâncreas $^{1,2,3,4}$. A pesquisa e a determinação dos reais dados epidemiológicos sobre tal lesão são prejudicadas pela vasta sinonímia envolvendo sua denominação ${ }^{3}$. No entanto,

1. Professor Assistente da Disciplina de Técnica Operatória e Cirurgia Experimental da UFRN. "Staff" da Cirurgia Oncológica da Liga Norte-Riograndense de Combate ao Câncer. Mestre em Cirurgia Gastroenterológica pela UFPE.

2. Estudante da Graduação do Curso Médico da UFRN.

3. Estudante da Graduação do Curso Médico da UFRN. Bolsista do CNPq.

Recebido em 05/7/2000

Aceito em 01/2/2001

Trabalho realizado na Disciplina de Técnica Operatória e Cirurgia Experimental da UFRN e na Liga Norte-Riograndense de Combate ao Câncer. 


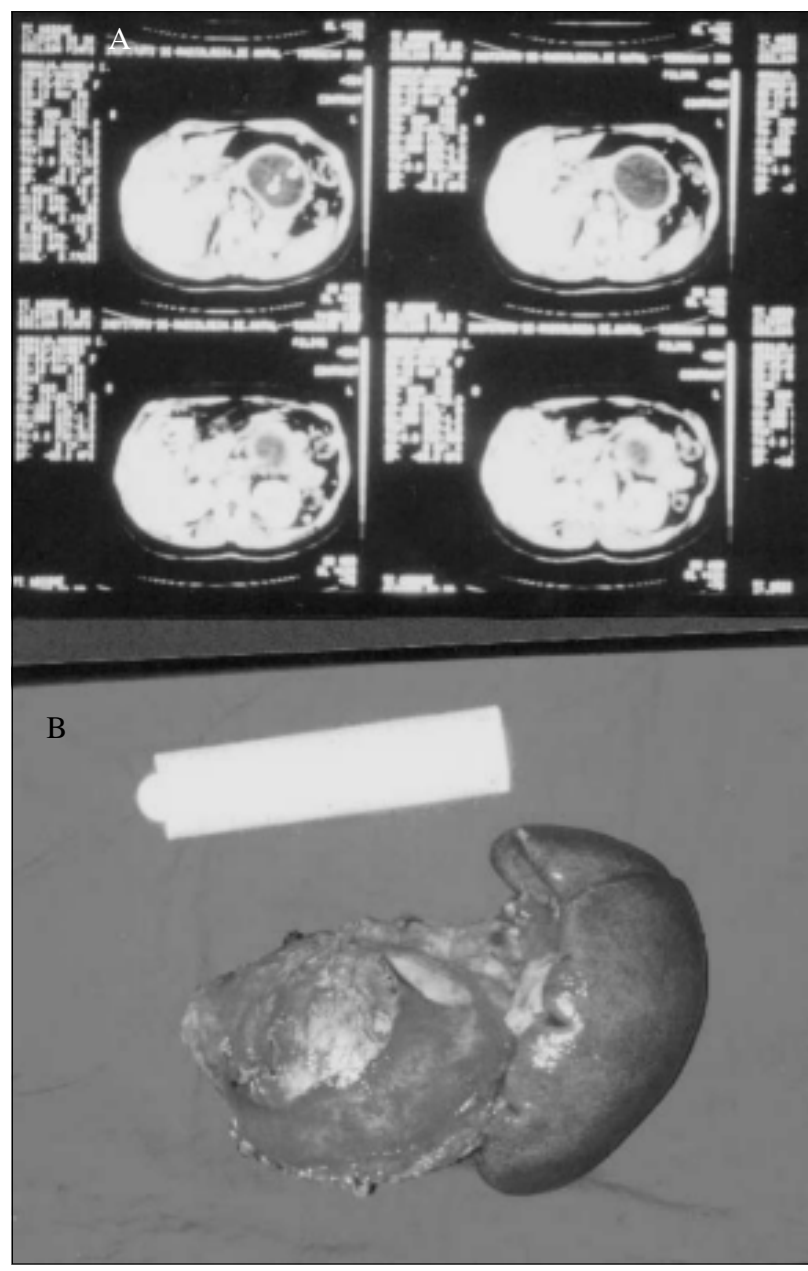

Figura $1-A$ : Cortes tomográficos evidenciando tumor sólidocístico em topografia do corpo e cauda do pâncreas. B: Peça operatória incluindo o baço.

está bem estabelecida a maior incidência entre mulheres jovens, com faixa etária média de 22 anos de idade ${ }^{2,5}$.

Essa predominância poderia sugerir a possibilidade de fatores hormonais terem influência na gênese desta neoplasia, todavia existem controvérsias sobre a presença ou não de receptores de estrógenos e progesterona nestes tumores ${ }^{3}$.

Possui apresentação variada, podendo o paciente ser completamente assintomático ou referir massa abdominal palpável, de crescimento lento, acompanhada ou não de dor abdominal aguda ou crônica, associada à epigastralgia ou a vômitos. Normalmente é diagnosticada por acaso em exames radiológicos para outras finalidades, como foi evidenciado no primeiro caso deste relato. Hemoperitôneo, devido à ruptura tumoral, e icterícia obstrutiva são incomuns pelo crescimento lento do tumor ${ }^{2,4,5}$.

$\mathrm{O}$ exame de imagem de maior acurácia no diagnóstico é a TC, que demonstra uma massa retroperitoneal, na topografia do pâncreas, de contornos bem definidos, encapsulada e lobulada, com áreas císticas e sólidas. São em geral grandes (em média, 10,3cm) e arredondadas e

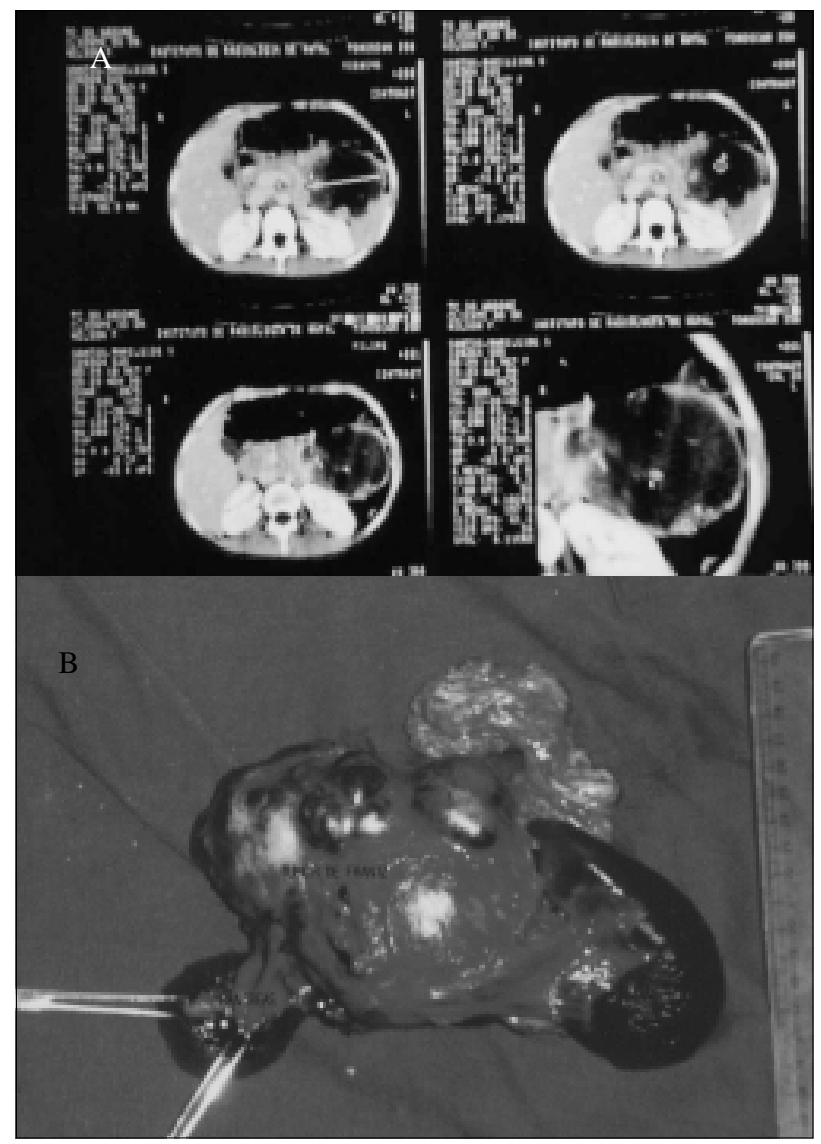

Figura 2 - A: Corte tomográfico (lesão em corpo-cauda do pâncreas). B: Pancreatectomia corpo-caudal associada à esplenectomia, devido à aderência tumoral aos vasos esplênicos.

localizam-se preferencialmente no corpo e cauda do pâncreas $(64 \%)$. A angiografia mostra pouco ou nenhum suprimento vascular para o tumor ${ }^{2,5}$.

A citologia do aspirado por punção guiada por ultra-sonografia pode também ajudar no diagnóstico ${ }^{4,5}$.

A microscopia exibe, freqüentemente, formações papilares com células de tamanho uniforme e citoplasma levemente eosinofílico, ao redor de um eixo fibroconjuntivo, de qualidade mixomatosa (importante para o diagnóstico) e mitoses incomuns; por outro lado, na área sólida, há degeneração de grupos celulares. O tumor é contido regularmente por cápsula fibrosa verdadeira, com pequenos focos infiltrativos, que não costumam se estender ao parênquima pancreático ${ }^{3,4}$. Tais características foram evidenciadas nos casos ora relatados.

O tumor exibe grande crescimento local, sendo raras a invasão extrapancreática e as metástases a distância. Apesar da marcada tendência à benignidade, uma vez diagnosticada a neoplasia, não existe justificativa para manter conduta expectante como foi proposto para a primeira paciente. $\mathrm{O}$ acometimento do fígado, pulmão e pele é excepcional, porém quando presente é mais comum em mulheres idosas ${ }^{2,3,5}$. 
O tratamento de eleição é a ressecção cirúrgica completa do tumor, sendo esta usualmente curativa. Se a lesão estiver situada no corpo ou na cauda do pâncreas a pancreatectomia distal com preservação esplênica deve ser realizada ${ }^{2,4}$. Nas duas pacientes a esplenectomia foi indicada devido à aderência que existia do processo tumoral com os vasos esplênicos. Para os tumores localizados na cabeça do pâncreas deve-se realizar a duodenopancreatectomia com preservação pilórica ${ }^{2}$.

Nos casos irressecáveis pode-se realizar a drenagem interna (cistojejunostomia em Y de Roux), radioterapia ou quimioterapia 5 .

\begin{abstract}
Papyllary cystic tumor of the pancreas, so-called Frantz's tumor, is rare. Clinical presentation of this disease is usually a slowly growing abdominal mass with or without abdominal pain, affecting predominantly young females. Its pathogenesis is still unknown. Surgical resection is usually curative, and prognosis is excellent. The authors report two pancreatic tumor cases(Frantz's tumor) in women aged 26 and 31 years old. Pre operative assessment showed a solid-cystic tumor of the tail and body of the pancreas. An extended distal pancreatectomy was performed without splenic preservation.
\end{abstract}

Key Words : Frantz's tumor; Pancreatic tumor.

\section{REFERÊNCIAS}

1. Frantz VK. Tumors of the pancreas. In: Atlas of tumor pathology section VII, Fascicles 27 and 28. Washington (DC): Armed Forces Institute of Pathology, 1959.

2. Pereira Jr GA, Stracieri LDS, Ceneviva R et al. Neoplasia papilar cístico-sólida de pâncreas. Rev Col Bras Cirur 1998; 25(5): 351-353.

3. Machado MCC, Cunha JEM, Bacchella T, Jukemura J et al. Tumor de Frantz (neoplasia papilar e cística do pâncreas): estudo de três casos. Rev Hosp Clín Fac Med 1993; 48(1): 29-34.

4. Yang YJ, Chen JS, Chen CJ, Lin PW et al. Papillary cystic tumor of the pancreas in children. Scand J Gastroenterol 1996; 31: 1223-1227.
5. Horisawa M, Niinomi N, Sato T, Yokoi S et al. Frantz's tumor (solid and cystic tumor of the pancreas) with liver metastasis: successful treatment and long-term follow-up. J Ped Surg 1995; 30(5): 724-726.

Endereço para correspondência:

Dr. Francisco Edilson Leite Pinto Júnior

Av. Brigadeiro Gomes Ribeiro, 1025

59056-520 - Natal-RN

E-mail: edilsonpinto@uol.com.br 